\title{
A GENERALIZATION OF THE PUNCTURED NEIGHBORHOOD THEOREM
}

\author{
WOO YOUNG LEE
}

(Communicated by Palle E. T. Jorgensen)

\begin{abstract}
If $T \in \mathscr{L}(X)$ is regular on a Banach space $X$, with finite- dimensional intersection $T^{-1}(0) \cap T(X)$, and if $S, S^{\prime}$ are invertible, commute with $T$ and have sufficiently small norm, then $\operatorname{dim}\left(T-S^{\prime}\right)^{-1}(0)=\operatorname{dim}(T-S)^{-1}(0)$ and $\operatorname{dim} X /\left(T-S^{\prime}\right) X=\operatorname{dim} X /(T-S) X$.
\end{abstract}

In [5], Lee proved that if $T$ is a regular operator with some finite-dimensional intersection property on a Banach space and if 0 is the boundary of the spectrum of $T$, then 0 is an isolated point of the spectrum of $T$.

In this note we derive a generalization of the punctured neighborhood theorem and then strengthen the above result.

Throughout this note suppose $X$ and $Y$ are complex Banach spaces, write $\mathscr{L}(X, Y)$ for the set of bounded linear operators from $X$ to $Y$, and abbreviate $\mathscr{L}(X, X)$ to $\mathscr{L}(X)$. If $T \in \mathscr{L}(X)$ then we write $\sigma(T)$ for the spectrum of $T$. If $K$ is a compact subset of the complex plane $\mathbf{C}$, write $\partial K$ and iso $(K)$, respectively, for the topological boundary points and the isolated points of $K$.

We recall that $T \in \mathscr{L}(X, Y)$ is said to be bounded below if there is $k>0$ for which $\|x\| \leq k\|T x\|$ for all $x \in X$ and is said to be regular if there is $T^{\prime} \in \mathscr{L}(Y, X)$ for which $T=T T^{\prime} T$. It is known that $T$ is regular if and only if $T(X)$ is closed and both $T^{-1}(0)$ and $T(X)$ are complemented and that

$$
T \text { regular and one-one } \Rightarrow T \text { bounded below } \Rightarrow T(X) \text { closed }
$$

(cf. [2, 3]). Recall, also, that $T \in \mathscr{L}(X, Y)$ is said to be Fredholm if $T^{-1}(0)$ and $Y / T(X)$ are finite dimensional. If $T \in \mathscr{L}(X, Y)$ is Fredholm then the index of $T$ is defined by

$$
\operatorname{index}(T)=\operatorname{dim} T^{-1}(0)-\operatorname{dim} Y / T(X) .
$$

If $T \in \mathscr{L}(X)$ then the hyperrange of $T$ is the subspace

$$
T^{\infty}(X)=\bigcap_{n=1}^{\infty} T^{n}(X) .
$$

We begin with a modification of [2, Theorem 7.8.3]:

Received by the editors May 9, 1991.

1991 Mathematics Subject Classification. Primary 47A10.

Key words and phrases. Punctured neighborhood theorem, regular operators, spectrum.

Research supported in part by a grant from ADD in 1991. 
Lemma 1. Let $X$ be a normed space and $T \in \mathscr{L}(X)$. If the intersection $T^{-1}(0) \cap T^{k}(X)$ is finite dimensional for some $k$ then

$$
T\left(T^{\infty}(X)\right)=T^{\infty}(X) .
$$

If $S \in \mathscr{L}(X)$ is invertible and commutes with $T$, then

$$
(T-S)^{-1}(0) \subseteq T^{\infty}(X) .
$$

Proof. The proof of equality (1.1) is taken straight from a slight modification of the proof of $[2,(7.8 .3 .2)]$, which works with the stronger assumption $\operatorname{dim} T^{-1}(0)<\infty$. The inclusion (1.2) is just the inclusion [2, (7.8.3.4)].

Our main theorem is a generalization of the "punctured neighborhood theorem."

Theorem 2. If $T \in \mathscr{L}(X)$ is regular on a Banach space $X$, with finite-dimensional intersection $T^{-1}(0) \cap T(X)$, and if $S, S^{\prime}$ are invertible, commute with $T$ and have sufficiently small norm, then

$$
\operatorname{dim}\left(T-S^{\prime}\right)^{-1}(0)=\operatorname{dim}(T-S)^{-1}(0)
$$

and

$$
\operatorname{dim} X /\left(T-S^{\prime}\right) X=\operatorname{dim} X /(T-S) X .
$$

Proof. Suppose $T \in \mathscr{L}(X)$ is regular and $T^{-1}(0) \cap T(X)$ is finite dimensional. We begin by showing $T^{\infty}(X)$ is complete. To do this, define $S_{1}: X / T^{-1}(0) \rightarrow$ $X$ by setting

$$
S_{1}\left(x+T^{-1}(0)\right)=T x \in X \quad \text { for each } x \in X .
$$

Then, by (0.1) $S_{1}$ is bounded below. Our assumption also gives (with the aid of [5, Lemma 1]) that the subspace $T(X)+T^{-1}(0)$ is closed in $X$. Further, we can find a closed subspace $W \subseteq T(X)$ for which

$$
T(X)+T^{-1}(0)=W+T^{-1}(0) \quad \text { and } \quad T(X)=W \oplus\left(T^{-1}(0) \cap T(X)\right) .
$$

We can then regard $W+T^{-1}(0)=\left\{w+T^{-1}(0): w \in W\right\}$ as a closed subspace of $X / T^{-1}(0)$. If we define $S_{2}: W+T^{-1}(0) \rightarrow X$ by setting

$$
S_{2}\left(w+T^{-1}(0)\right)=T w \in X \quad \text { for each } w \in W,
$$

then $S_{2}$ is also bounded below (see [2, (3.11.1.2)]). Since $W+T^{-1}(0)$ is complete, it follows from $(0.1)$ that $S_{2}\left(W+T^{-1}(0)\right)=T(W)=T^{2}(X)$ is closed in $X$. Inductively, we have that $T^{n}(X)$ is closed in $X$ for each $n \in N^{T}$, hence so is $T^{\infty}(X)$; therefore, $T^{\infty}(X)$ is complete. We write $U^{\wedge}: T^{\infty}(X) \rightarrow$ $T^{\infty}(X)$ for the operator induced by $U \in \operatorname{comm}(T)$, where $\operatorname{comm}(T)$ is the "commutant" of $T$ in $\mathscr{L}(X)$. Then, since $\left(T^{\wedge}\right)^{-1}(0)=T^{-1}(0) \cap T^{\infty}(X) \subseteq$ $T^{-1}(0) \cap T(X)$ and, by (1.1), $T^{\wedge}$ is onto, it follows that $T^{\wedge}$ is Fredholm. If $S$ has sufficiently small norm then $(T-S)^{\wedge}$ is also Fredholm because the Fredholm operators on a Banach space form an open set. We now claim that

$$
\operatorname{dim}(T-S)^{-1}(0)=\operatorname{dim}(T-S)^{\wedge^{-1}}(0)=\operatorname{index}(T-S)^{\wedge}=\operatorname{index}\left(T^{\wedge}\right) .
$$

The first equality comes from (1.2), the second equality comes from the fact that, by the first equality and $(1.1),(T-S)^{\wedge}$ is onto, and the third equality comes from the continuity of the Fredholm index. Since the right-hand side of 
(2.3) is independent of $S$, equality (2.1) follows. Also applying the "classical" punctured neighborhood theorem of $T-S$ gives the equality (2.2).

Our proof of Theorem 2 closely follows the original argument of Harte [2, Theorem 7.8.4], which assumes $T$ is Fredholm.

The following result is an improvement of [5, Theorem 2]:

Corollary 3. If $T \in \mathscr{L}(X)$ is regular on a Banach space $X$, with finite-dimensional intersection $T^{-1}(0) \cap T(X)$, then there is implication

$$
0 \in \partial \sigma(T) \Rightarrow 0 \in \text { iso } \sigma(T) \text {. }
$$

Proof. Apply Theorem 2 to $T-S$ with $S=\mu I$ and $0<|\mu|<\varepsilon$; then $\operatorname{dim}(T-\lambda I)^{-1}(0)$ and $\operatorname{dim} X /(T-\lambda I) X$ are constant on a punctured neighborhood of 0 . If $0 \in \partial \sigma(T)$ then it follows that for some $\theta$ with $0<\theta<\varepsilon$, $\operatorname{dim}(T-\lambda I)^{-1}(0)=\operatorname{dim} X /(T-\lambda I) X=0$ for $0<|\lambda|<\theta$, which says that $0 \in$ iso $\sigma(T)$.

Recall that $T \in \mathscr{L}(X)$ is said to be relatively almost open if its truncation $\widehat{T}: X \rightarrow T(X)$ is almost open (cf. [2, 4]). If $T \in \mathscr{L}(X)$ for a Banach space $X$ then by the open mapping theorem we have

$$
T \text { relatively almost open } \Leftrightarrow T(X) \text { closed. }
$$

In the context of a Hilbert space we can simplify Corollary 3 . In a sense, the following result is an improvement of [6, Theorem 1].

Corollary 4. If $X$ is a Hilbert space and $T \in \mathscr{L}(X)$ is relatively almost open, with finite-dimensional intersection $T^{-1}(0) \cap T(X)$, then there is implication

$$
0 \in \partial \sigma(T) \Rightarrow 0 \in \text { iso } \sigma(T) \text {. }
$$

Proof. If $T \in \mathscr{L}(X)$ for a Hilbert space $X$ then both $T^{-1}(0)$ and $\operatorname{cl} T(X)$ are always complemented; thus we have

$$
T \text { regular } \Leftrightarrow T(X) \text { closed; }
$$

therefore, (3.1) together with (3.2) and (4.2) gives (4.1).

\section{ACKNOWLEDGMENT}

The author would like to express his thanks to the referee whose suggestions led to an improvement of the paper.

\section{REFERENCES}

1. S. Goldberg, Unbounded linear operator, McGraw-Hill, New York, 1966.

2. R. Harte, Invertibility and singularity for bounded linear operators, Marcel Dekker, New York, 1988.

3. H. G. Heuser, Functional analysis, Wiley, New York, 1982.

4. W. Y. Lee, Relatively open mappings, Proc. Amer. Math. Soc. 108 (1990), 93-94.

5. __ Boundaries of the spectra in $\mathscr{L}(X)$, Proc. Amer. Math. Soc. 116 (1992), 185-189.

6. C. R. Putnam, The spectra of operators having resolvents of first order growth, Trans. Amer. Math. Soc. 133 (1968), 505-510. 